\title{
The Journal of Applied \& Computational Mathematics (JACM)
}

\section{Kinkar Ch. Das*}

Department of Mathematics, College of Science, Sungkyunkwan University

The Journal of Applied \& Computational Mathematics was established in the year 2012. It makes publicly available the most significant technical and scientific developments, and research in the field of applied and computational mathematics. The JACM journal is an open access publication model that enables the dissemination of research articles to the global community and thus can be accessed by anyone.

The digital articles are delivered electronically to your device. You can listen to your research almost immediately, at any time of the day or night and listening makes it more exciting and easier to focus, reducing the hours of starring at the monitors. This will continue to be more beneficial with fast evolving technologies on electronic books. It's available in more than 50 languages giving you the choice of your interest to read the articles, which enables worldwide communication with researchers in a variety of languages.

Open access is an important step on the way towards open science. We observe the open access day (OA Day) which strives to raise awareness amongst researchers, academic publishers, research funders, students and the public the importance of open access for our global society. With this awareness there arises a few questions like; why should scientific and mathematical research be an open access resource to this world? OA marks a turning point within the scientific cycle. This communication step is crucial, as it adds to our global knowledge foundation for new research questions or ideas that may eventually lead to things like "innovation", "insight" and "progress". Knowledge grows when shared. What else is the goal of research if not growing knowledge on a global scale? It would allow to more adequately evaluating the research accomplishment of young scientists.

Now how open can science and research be? If the research is scientific in nature, it is frequently referred to as open science. It is online, free of charge and free of unnecessary copyright and licensing restrictions supporting wider and faster access to knowledge. This result in a revolutionary opportunity to accelerate research and share knowledge, especially important for researchers and educators in developing countries where access to knowledge has been sharply reduced by four decades of fast rising journal prices.
We all know and believe that the process of creating open-source products involves the iterative cycle of (1) a need or problem being identified, (2) a preliminary solution being posted to this problem, (3) an open appeal to the wider community being made, (4) inputs received from an unrestricted community and (5) the cycle beginning over again. Such a cycle can operate quickly because of the advent of online tools that strengthen the relevant networks.

Being a part of the OMICS group I am enjoying and playing around with the platforms and technologies that may facilitate the transition to a more open scientific cycle, keeping a special eye on what these upcoming changes might mean to young scientists. It set a foundation for my future work as an author, reviewer and an editor. Others can, of course, familiarize themselves with the issue of effectively (in both resource and time) communicating research results via the channels that are technically possible. They can experiment with the tools at hand to communicate their thoughts, and they can educate even more others about these matters in more traditional ways. The journal allows sharing your views regarding its research articles on social networking sites like twitter, linkedin etc. giving space to individual thoughts. Open science is a research accelerator.

\section{Conclusion}

I am thankful to the OMICS group and other supporting members who gave me a heart to greatly enjoy the collaboration team work in publishing this journal. We hope heartily to see that with the dedication of capable staff (5000 editorial team and 10,000 reviewers team), supporting organizations, with better international coverage (1 million readers) and a broader choice of topics, the journal would provide high levels of reliability and quality of service ( 21 day rapid review process, publication within 7 days of acceptance, quality and quick editorial, review). Thus although this Journal is based in U.S.A, through its authors, readers and editorial board it is essentially an international enterprise. We welcome thoughtful, concerned parenting from our varied readers and participants (in whatever way, it may be) for more continuity and structure and for whom we strive to make this a valuable one.
*Corresponding author: Kinkar Ch. Das, Department of Mathematics, College of Science, Sungkyunkwan University, Suwon 440-746, South Korea, E-mail: kinkar@lycos.com

Received April 09, 2012; Accepted April 12, 2012; Published April 15, 2012

Citation: Das CHK (2012) The Journal of Applied \& Computational Mathematics (JACM). J Applied Computat Mathemat 1:e108. doi:10.4172/2168-9679.1000e108

Copyright: (c) 2012 Das CHK. This is an open-access article distributed under the terms of the Creative Commons Attribution License, which permits unrestricted use, distribution, and reproduction in any medium, provided the original author and source are credited. 